\title{
INDICADORES MACRO E MICROMORFOLÓGICOS DA QUALIDADE FÍSICA DE UM LATOSSOLO VERMELHO TEXTURA MÉDIA CULTIVADO COM CANA-DE-AÇÚCAR NA MICRORREGIÃO DE QUIRINÓPOLIS, GOIÁS
}

\author{
MACRO AND MICROMORPHOLOGIC INDEX OF PHYSICAL RED LATOSOL \\ QUALITY OF MEDIUM TEXTURE CULTIVATED WITH SUGAR CANE IN \\ QUIRINÓPOLIS MICRO REGION, GOIÁS
}

\author{
INDICADORES MACRO Y MICROMORPHOLOGICO DE CALIDAD FÍSICA DE \\ UNO RED LATOSOL TEXTURA MEDIA CULTIVADO CON CAÑA DE AZÚCAR \\ EN LA MICRORREGIÓN QUIRINÓPOLIS, GOIÁS
}

\author{
Adriana Aparecida Silva - Universidade Estadual de Goiás - Cidade de Goiás - Goiás - Brasil \\ ueg.adriana@gmail.com
}

Selma Simões de Castro - Universidade Federal de Goiás - Goiânia - Goiás - Brasil

selma@iesa.ufg.br

\begin{abstract}
Resumo
A microrregião de Quirinópolis apresenta grandes áreas de cultivo de cana-de-açúcar sobre os melhores solos, os Latossolos Vermelhos distroférricos argilosos, e em virtude da demanda crescente expandiu-se sobre solos naturalmente menos férteis, como os Latossolos Vermelhos distróficos (LVd) de textura média. Tais solos são considerados frágeis do ponto de vista da estabilidade estrutural e da suscetibilidade a impactos, como a erosão e a compactação, em decorrência do manejo altamente tecnificado, como é o caso da cana-de-açúcar, que na região é cultivada com maquinário pesado em todas as fases do processo agrícola. 0 objetivo deste artigo é avaliar a qualidade física dos LVd cultivados com cana-de-açúcar, mas antes com outros usos (soja, pasto e sucessão pasto/soja), tendo como referência perfis de solo de área com vegetação remanescente. Para tanto, foram realizados estudos macro e micromorfológicos, físicos e de resistência mecânica à penetração. Os resultados revelaram mudanças na macro e microestrutura, consistência, dispersão de argila, densidade do solo e resistência à penetração nos perfis cultivados em comparação com o de vegetação natural, alterações compatíveis com degradação associada a processos de compactação, ainda que moderada.
\end{abstract}

Palavras-chave: Saccharum spp, estrutura, degradação, compactação.

\section{Abstract}

Quirinópolis Micro region depicts great expansion of sugar cane production on the clayed Red Latossols but due to the increasing demand of new areas the naturally less fertile, the dystrophic Red Latosol of medium texture was done. Such soils are considered weak to the extent of structural stability and impact installation such erosion and compression, this one primarily due to highly high technified management, as though the sugar cane, which heavy machinery during all stages of culture. The aim of this study was to evaluate the physical quality of LVd with current use of sugar cane, however previously varied successions (soybeans, pasture and grazing succession/soybean), with reference to soil profiles of areas with remaniant vegetation. Thus, we obtained macro and micromorphologic indicators, physical and penetration resistance. The results revealed changes in micro and macrostructure, consistency, clay dispersion, soil bulk density and penetration resistance on cultivated soil profiles compares with natural cover profile, indicators compatible of degradation by compaction, however moderate.

Key words: Saccharum spp, structure, degradation, compaction. 
Resumen

La microrregión de Quirinópolis tiene grandes áreas de cultivo de caña de azúcar en los mejores suelos, los Oxisoles dystroferric, debido a la creciente demanda, se amplió en suelos menos fértiles, como 0xisoles distróficos (LVd) textura media. Tales suelos son considerados débiles desde el punto de vista de la estabilidad estructural y la instalación de impactos como erosión y compactación, como resultado de esta gestión de la producción altamente intensiva, como es el caso de la caña de azúcar, que hace uso en la región de maquinaria pesado en todas las etapas de proceso agrícola. Nuestro objetivo fue evaluar la calidad física de los LVd con uso caña de azúcar, sino para otros usos (soya, pastos y sucesión pastoreo/soja), con referencia perfiles de suelo de área con vegetación remanente. Por lo tanto, hemos obtenido indicadores macro y micromorfológico, físicos y de la resistencia mecánicas. Los resultados revelaron cambios en la macro y microestructura, consistencia, dispersión de arcilla, densidad aparente del suelo y de resistencia a penetración en los perfiles cultivados en relación con el perfil cubierto por vegetación natural, cambios consistentes con la degradación asociada con los procesos de compresión, pero, moderada.

Palabras clave: Saccharum spp, estructura, degradación, compactación.

\section{Introdução}

A mesorregião Sul de Goiás apresenta a maior concentração de áreas de cultivo de cana-de-açúcar, estendendo-se, em 2012, por mais de 740 mil ha de área plantada, com destaque para as microrregiões Sudoeste, Meia Ponte, Quirinópolis e Vale do Rio dos Bois (Castro et al., 2010; Silva; Castro, 2011; Barbalho et al., 2013). O caso do processo de expansão da cana, na microrregião de Quirinópolis (MRQ), apresentou duas fases; a primeira entre 2004 e 2008, relativa a uma evidente substituição de áreas de culturas anuais, no caso a soja, concentrada na porção nordeste da microrregião, onde se localizam os melhores solos, os Latossolos Vermelhos distroférricos (LVdf). A partir de 2008, esgotada a área com melhores solos, passa a predominar a substituição de áreas de pastagens, localizadas a oeste e sul, sobre os demais solos, com maior expressão nos Latossolos Vermelhos distróficos (LVd) de textura média.

Os Latossolos Vermelhos se caracterizam por apresentar perfis homogêneos, associados a um relevo plano a suave ondulado. Dependendo da sua textura, são avaliados como solos altamente suscetíveis à compactação (quando apresentam textura média a argilosa ou muito argilosa), e dependendo do uso e manejo, sofrem subsequente degradação estrutural (Oliveira; Vaz; Reichardt, 1995; Oliveira et al., 2003). Os LVd, em particular, representam solos frágeis para o cultivo intensivo, em razão da sua textura média e do elevado teor de argila dispersa (Oliveira; Vaz; Reichardt, 1995). Por essas razões tais solos são considerados menos atraentes 
para o cultivo da cana-de-açúcar, mas, esgotadas as áreas com melhores solos, foram incorporados ao processo de expansão (Silva; Castro, 2011).

A compactação se refere à compressão do solo não saturado, promovendo a redução do volume dos poros com o rearranjo das partículas sólidas, ou seja, modificação da estrutura interna do solo (Dias Júnior, 2000). A compactação de solos cultivados com cana-de-açúcar tem sido atribuída ao manejo altamente tecnificado, devido ao uso de maquinaria pesada em todas as fases do cultivo (Pankhurst et al., 2003). Esse impacto ocorre com maior intensidade em solos argilosos (Oliveira et al., 2003), porém os solos de textura média a arenosos também revelam alta susceptibilidade à desagregação (Reichert et al., 2003; Severiano et al., 2009).

Dentre as propriedades físicas dos solos, a estrutura se destaca, uma vez que do ponto de vista do uso agrícola esse parâmetro é considerado o mais importante, por condicionar a porosidade do solo, influenciando fatores como suprimento de água e ar para as plantas (Schneider et al., 2007; Pedrotti; Méllo Júnior, 2009). Operações agrícolas como a atividade intensiva de preparo do solo, através de aração e gradagem, ou mesmo a mobilização de maquinário no cultivo e na colheita mecanizada, causam a quebra dos agregados e, consequentemente, das unidades estruturais, reduzindo seu tamanho e modificando o ambiente de crescimento radicular, como comumente observado em culturas de cana-de-açúcar (Roque et al., 2010; Pacheco; Cantalice, 2011).

Assim, para a avaliação da qualidade física do solo (QS), devem-se considerar os indicadores macro e micromorfológicos, em especial a estrutura e o comportamento, necessários para entender quais os possíveis impactos decorrentes do uso e manejo, como a mudança na forma, no arranjo e na frequência dos poros (morfoporosidade) e agregados (Castro, 1999). Na avaliação da estrutura do solo considera-se a perspectiva multiescalar. A macroscópica ou macromorfológica permite descrever, coletar amostras e realizar ensaios físico-hídricos nos perfis de solo em campo. Já a microscópica descreve as características micromorfológicas observáveis com equipamento ótico, com aumento $\geq 5 \mathrm{X}$ em microscópio ótico, sendo indispensável relacionar esses resultados com aqueles da escala anterior (Castro et al., 2003).

Neste estudo, apresenta-se uma avaliação dos indicadores macro e micromorfológicos da qualidade física dos LVd da microrregião de Quirinópolis (MRQ), a fim de se discutir a compactação e a subsequente ins- 
tabilidade estrutural de perfis de solos cultivados com cana-de-açúcar a partir de 2008. Os perfis de solos serão comparados correlacionando-os ao histórico de substituição de uso soja/cana, pasto/cana e sucessão pasto/ soja/cana, considerado como referência um perfil com vegetação natural. As avaliações macro e micromorfológicas são acompanhadas de resultados analíticos da consistência, textura, argila dispersa em água (ADA), grau de floculação (GF), densidade do solo (Ds) e de partícula (Dp), porosidade total (PT) e resistência mecânica à penetração (RP).

\section{Materiais e métodos}

A MRQ, composta por nove municípios, possui uma área total de $16.068,103 \mathrm{~km}^{2}$ e se localiza na região de planejamento denominada Sudoeste de Goiás. Quirinópolis e Gouvelândia se destacam no processo de expansão da cana-de-açúcar. Os LVd se situam a oeste e sul da microrregião, sendo originários dos arenitos da Formação Rio do Peixe, associados à Superfície Regional de Aplainamento IIIB, com cotas entre 650 e 750 $\mathrm{m}$, dissecação média e declividades predominantes entre 3\% e 6\% (Latrubesse; Carvalho, 2006). Até recentemente, nestes solos, predominava a pastagem extensiva, que cobria mais de $72 \%$ da área, seguida pela cultura de grãos (soja) em menos de 10\% da área. Atualmente, esse uso mudou, houve a conversão para o cultivo de cana-de-açúcar, que em 2010 ocupava cerca de 7\% da área (Silva; Castro, 2011).

Para este estudo, foram selecionadas três áreas relativas a talhões de cana-de-açúcar no $4^{\circ}$ corte do $1^{\circ}$ ciclo, plantadas com a mesma variedade de cana (SP 813250), com manejo sem queima ou fertirrigação, cultivo e colheita fortemente mecanizados e o mesmo preparo inicial do solo (subsolagem, grade pesada, calagem, gessagem, fosfatagem, grade leve). Cada uma dessas áreas apresenta substituição de culturas habituais na MRQ, sendo soja/cana, pasto/cana e sucessão pasto/soja/cana. Como referência, foi selecionada uma quarta área com vegetação natural (Cerrado). Todos os pontos apresentam características homogêneas em relação à geologia, geomorfologia, declividade e aos solos. O trabalho de campo foi realizado em novembro de 2010, no período de estação úmida.

Em campo foram realizadas descrições morfológicas em trincheiras convencionais (Santos et al., 2005), enfatizando-se a espessura, textura, estrutura, consistência e porosidade, além de observada a presença 
de processo de compactação, suas características e seus efeitos. Foram realizadas coletas de amostras deformadas para análises físicas e de fertilidade (amostras compostas) e indeformadas (anéis volumétricos para densidade e caixas retangulares de papel-cartão branco, tamanho 12x7x6 $\mathrm{cm}$, em que foram moldados monólitos com estrutura de campo preservada, para o estudo micromorfológico). As amostras são relativas a cada um dos horizontes dos quatro perfis de solo analisados.

Ao lado das trincheiras, houve testes de resistência mecânica à penetração do solo (RP), que simula a penetração das raízes nos solos feita por meio do penetrômetro vertical de impacto $(60 \mathrm{~cm})$, modelo IAA/Planalçucar (Stolf et al., 1983). A transformação da penetração da haste do aparelho no solo (cm/impacto), em valores de RP, foi obtida pela equação: $\mathrm{R}\left(\mathrm{kgf} / \mathrm{cm}^{2}\right)=5,6+6,89 \mathrm{~N}$, em que: $\mathrm{R}=$ resistência do solo ou índice do cone; e $\mathrm{N}$ = penetração unitária por um impacto. Para a conversão de kgf/ $\mathrm{cm}^{2}$ para $\mathrm{MPa}$, multiplicou-se o resultado obtido na equação pela constante 0,0981 .

Em laboratório, as amostras deformadas destinaram-se à análise textural (em água e $\mathrm{NaOH}+$ Hexametafosfato de Na pelo método do densímetro), e também às análises de umidade gravimétrica, ADA, GF, MO (Dicromato de Na). Já as amostras indeformadas (anéis volumétricos) serviram à determinação da Ds (método do anel volumétrico), Dp (método do balão volumétrico) e cálculo da PT (EMBRAPA, 1997).

As amostras indeformadas do tipo monólitos foram secas em estufa a $40^{\circ} \mathrm{C}$ e impregnadas sob vácuo com resina de poliéster para posteriormente serem cortadas, coladas e polidas até alcançarem a espessura de 25 $\mu \mathrm{m}$ (Castro et al., 2003). Os parâmetros micromorfológicos observados foram trama, fundo matricial, microestrutura, porosidade dominante e feições pedológicas dominantes. As descrições foram feitas conforme Stoops (2003), com o auxílio de microscópio escarificação binocular da Olympus BX40, acoplado a uma máquina digital para a tomada de fotomicrografias.

\section{Resultados e discussão}

\section{Indicadores macromorfológicos}

Os perfis estudados obedeceram à numeração de campo: 3B (soja/ cana), 4B (pasto/cana), 5B (sucessão pasto/soja/cana) e 8C, perfil de referência (vegetação natural). O manejo foi plantio sobre palhada e adubação 
convencional; os perfis de solos foram preparados da mesma maneira antes do primeiro plantio (escarificação, grade pesada, grade leve, correção e adubação), o que teria reduzido ou eliminado o efeito dos usos e manejos anteriores, logo de eventual compactação herdada (Dias Júnior, 2000).

De um modo geral, constatou-se certa homogeneidade macroestrutural dos solos analisados, corroborando as características conhecidas desse tipo de solo (EMBRAPA, 2006). Nesse sentido, predomina a estrutura em blocos subangulares pequenos que se desfazem em granular pequena a muito pequena em todos os horizontes dos perfis atualmente com cana-de-açúcar, a saber, 3B, 4B e 5B, exceto os horizontes A dos perfis com cultura, que apresentaram blocos subangulares médios (Quadro 1).

Quadro 1 - Atributos morfológicos dos Latossolos Vermelhos distróficos.

\begin{tabular}{|c|c|c|c|c|c|}
\hline \multirow{2}{*}{$\begin{array}{c}\text { Horizonte } \\
\mathrm{Cm}\end{array}$} & \multirow[t]{2}{*}{ Estrutura } & \multicolumn{3}{|c|}{ Consistência } & \multirow[t]{2}{*}{ Transição } \\
\hline & & Seco & Úmido & Molhado & \\
\hline \multicolumn{6}{|c|}{ PERFIL 3B - soja/cana } \\
\hline A $0-30$ & Blocos subangulares médios & $\mathrm{S}$ & FR & LPL, LPE & $\mathrm{D}, \mathrm{O}$ \\
\hline $\mathrm{AB} 30-70$ & Blocos subangulares pequenos & $\mathrm{D}$ & FI & LPL, PE & $\mathrm{D}, \mathrm{P}$ \\
\hline BA $70-97$ & Blocos subangulares pequenos & $\mathrm{D}$ & FR & LPL, PE & $\mathrm{C}, \mathrm{P}$ \\
\hline Bw1 $97-118$ & Blocos subangulares pequenos & $\mathrm{D}$ & FR & LPL, LPE & $\mathrm{C}, \mathrm{P}$ \\
\hline Bw2 $118-150+$ & Blocos subangulares pequenos & $\mathrm{D}$ & FR & LPL, LPE & \\
\hline \multicolumn{6}{|c|}{ PERFIL 4B - pasto/cana } \\
\hline A $0-20$ & Blocos subangulares médios & $\mathrm{S}$ & FR & LPL, LPE & $\mathrm{G}, \mathrm{O}$ \\
\hline AB $20-44$ & Blocos subangulares pequenos & MD & FI & LPL, LPE & $\mathrm{C}, \mathrm{O}$ \\
\hline BA $44-72$ & Blocos subangulares pequenos & $\mathrm{D}$ & FI & LPL, LPE & $\mathrm{C}, \mathrm{P}$ \\
\hline Bw1 $72-119$ & Blocos subangulares pequenos & $\mathrm{D}$ & FR & LPL, LPE & $\mathrm{C}, \mathrm{P}$ \\
\hline Bw2 $119-150+$ & Blocos subangulares pequenos & $\mathrm{D}$ & FR & LPL, LPE & \\
\hline \multicolumn{6}{|c|}{ PERFIL 5B - sucessão pasto/soja/cana } \\
\hline A $0-29$ & Blocos subangulares médios & $\mathrm{D}$ & FI & LPL, LPE & $\mathrm{G}, \mathrm{O}$ \\
\hline AB $29-56$ & Blocos subangulares pequenos & MD & FI & LPL, LPE & $\mathrm{C}, \mathrm{O}$ \\
\hline BA $56-73$ & Blocos subangulares pequenos & $\mathrm{LD}$ & FR & NPL, NPE & $\mathrm{C}, \mathrm{P}$ \\
\hline Bw1 $73-97$ & Blocos subangulares pequenos & LD & FR & LPL, LPE & $\mathrm{C}, \mathrm{P}$ \\
\hline Bw2 $97-150+$ & Granular pequena & LD & FR & LPL, LPE & \\
\hline \multicolumn{6}{|c|}{ PERFIL 8C - vegetação natural } \\
\hline A $0-13$ & Blocos subangulares pequenos & $\mathrm{S}$ & FR & PL, LPE & $\mathrm{C}, \mathrm{P}$ \\
\hline $\mathrm{AB} 13-41$ & Blocos subangulares pequenos & LD & FR & LPL, LPE & $\mathrm{C}, \mathrm{P}$ \\
\hline BA $41-60$ & Blocos subangulares pequenos & LD & FR & LPL, LPE & $\mathrm{A}, \mathrm{P}$ \\
\hline Bw1 $60-88$ & Blocos angulares pequenos & LD & FR & LPL, NPE & $\mathrm{C}, \mathrm{P}$ \\
\hline Bw2 $88-150+$ & Blocos angulares pequenos & $\mathrm{M}$ & FR & LPL, LPE & \\
\hline
\end{tabular}

Consistência seca: $\mathrm{S}=$ solta, $\mathrm{M}=$ macia, $\mathrm{LD}=$ ligeiramente dura, $\mathrm{MD}=$ dura, $\mathrm{M}=$ muito dura; Consistência úmida: $\mathrm{FR}=$ friável,

$\mathrm{FI}=$ firme; Consistência molhada: $\mathrm{NPL}=$ não plástica, $\mathrm{LPA}=$ ligeiramente plástica, $\mathrm{PL}=$ plástica, $\mathrm{NPE}=$ não pegajosa, $\mathrm{LPE}=$ ligeiramente pegajosa, $\mathrm{PE}=$ pegajosa; Transição: $\mathrm{A}=$ abrupta, $\mathrm{C}=$ clara, $\mathrm{G}=$ gradual, $\mathrm{D}=$ difusa, $\mathrm{P}=$ plana, $\mathrm{O}=$ ondulada. 
A consistência seca se apresentou dura de modo relativamente constante em profundidade e ao longo dos perfis com cana-de-açúcar, com exceção do horizonte A dos perfis 3B, 4B e 8C, em que é solta, e do perfil $5 \mathrm{~B}$, que passa a muito dura e ligeiramente dura em profundidade. $\mathrm{O}$ perfil 8C apresentou consistência seca solta no horizonte A, ligeiramente dura nos horizontes AB, BA e Bw1, e macia no horizonte Bw2, comportando-se como uma massa de solo fracamente coerente que se desfaz em granular pequena.

A consistência úmida desses solos apresentou-se firme nos horizontes $\mathrm{AB}$ de todos os perfis com cultivo, além dos horizontes A do perfil $5 \mathrm{~B}$ e BA do perfil 4B. Nos demais horizontes de todos os perfis, a consistência úmida é friável, revelando que a dureza é sazonalmente reversível. Em relação à consistência molhada, esta se apresentou, no geral, ligeiramente plástica e pegajosa em todos os horizontes de todos os perfis, sendo pegajosa nos horizontes $\mathrm{AB}$ e $\mathrm{BA}$ do perfil 3B, não plástica e não pegajosa no horizonte BA do perfil 5B e plástica no horizonte A do perfil 8C.

A consistência seca dura a muito dura é indicadora de compactação, característica observada nos horizontes superficiais dos perfis 3B (exceto no horizonte A), 4B e 5B. Nos horizontes profundos do perfil 8C predomina a consistência ligeiramente dura. Contudo, o fato de se mostrar friável, quando úmida, indica a sua não compactação, o que não ocorre nos horizontes $\mathrm{AB}$ dos perfis 3B, 4B e 5B, em que é firme, uma característica de horizonte compactado. É importante lembrar que esses solos passaram por processo de preparo que teria reduzido ou eliminado o efeito dos usos e manejos anteriores (Dias Júnior, 2000). No entanto, esse preparo ocorreu no ano de 2006, após o que já foram realizadas três colheitas mecanizadas (junho/2008, maio/2009 e maio/2010), o que permite supor que a colheita poderia ser a provável causa da mudança estrutural e, consequentemente, da alteração destes solos, conforme também observado por Casagrande (2002).

Em relação aos atributos físicos e ao teor de matéria orgânica (Tabela 1), os dados revelam diferenciação textural entre os horizontes superficiais e subsuperficiais: no A, AB e BA, a textura é franco-argilo-arenosa e nos horizontes Bw1 e Bw2 é argilo-arenosa, exceto no perfil 3B, de textura franco-argilo-arenosa em todo o perfil. Esse aumento da fração argila nos horizontes subsuperficiais é esperado para solo de textura média como o LVd (EMBRAPA, 2006). Nesse sentido, a redução da fração argila 
nos horizontes superficiais $\mathrm{A}$ e $\mathrm{AB}$ de todos os perfis, inclusive para o perfil 8C, acompanhada pelo aumento dos valores de ADA, é própria desse tipo de solo, e não necessariamente decorrente do manejo, dado que o 8C também apresenta o mesmo comportamento.

Foram observados valores elevados de Ds para todos os horizontes A, de todos os perfis $(>1,63)$, sendo que no perfil $5 B$ chega a 1,83 $\mathrm{g} \mathrm{cm}^{-3}$ no horizonte A, considerado como valor crítico. Valores de Ds entre 1,40 a 1,50 $\mathrm{g} \mathrm{cm}^{-3}$ nos solos de textura média, são atribuídos frequentemente a algum grau de compactação (Reichert et al., 2003). Paralelamente, os valores de GF se apresentaram menores nos horizontes superficiais, indicando uma possível instabilidade estrutural nesses horizontes.

Tabela 1 - Atributos físicos e teor de matéria orgânica do Latossolo Vermelho distrófico.

\begin{tabular}{|c|c|c|c|c|c|c|c|c|c|c|}
\hline \multirow{2}{*}{$\begin{array}{c}\text { Horizonte } \\
\mathrm{Cm}\end{array}$} & \multicolumn{3}{|c|}{$\begin{array}{c}\text { Textura } \\
\mathrm{g} \mathrm{Kg}^{-1}\end{array}$} & \multirow{2}{*}{ Classe textural } & \multirow{2}{*}{$\begin{array}{c}\text { ADA } \\
\%\end{array}$} & \multirow{2}{*}{$\begin{array}{c}\text { GF } \\
\%\end{array}$} & \multirow{2}{*}{\begin{tabular}{|c|} 
Densidade \\
Solo \\
$\mathrm{g} \mathrm{cm}^{-3}$
\end{tabular}} & \multirow[b]{2}{*}{\begin{tabular}{|l|} 
Part. \\
$\mathrm{g} \mathrm{cm}^{-3}$
\end{tabular}} & \multirow{2}{*}{$\begin{array}{l}\text { PT } \\
\%\end{array}$} & \multirow{2}{*}{$\begin{array}{c}\text { MO } \\
\mathrm{g} \mathrm{cm}^{-3}\end{array}$} \\
\hline & Areia & Silte & Argila & & & & & & & \\
\hline \multicolumn{11}{|c|}{ PERFIL 3B - soja/cana não fertirrigada } \\
\hline A $0-30$ & 640 & 80 & 280 & Franco-argilo-arenoso & 12 & 60 & 1,69 & 2,46 & 31 & 17 \\
\hline $\mathrm{AB} 30-70$ & 620 & 80 & 300 & Franco-argilo-arenoso & 12 & 65 & 1,51 & 2,20 & 32 & 9 \\
\hline BA $70-97$ & 570 & 90 & 340 & Franco-argilo-arenoso & 0 & 100 & 1,52 & 2,60 & 41 & 6 \\
\hline Bw1 $97-118$ & 570 & 90 & 340 & Franco-argilo-arenoso & 2 & 94 & 1,43 & 2,56 & 44 & 3 \\
\hline Bw2 $118-150+$ & 570 & 90 & 340 & Franco-argilo-arenoso & 0 & 100 & 1,43 & 2,67 & 46 & 2 \\
\hline \multicolumn{11}{|c|}{ PERFIL 4B - pasto/cana não fertirrigada } \\
\hline A $0-20$ & 590 & 90 & 320 & Franco-argilo-arenoso & 8 & 78 & 1,64 & 2,38 & 31 & 10 \\
\hline AB $20-44$ & 580 & 90 & 330 & Franco-argilo-arenoso & 4 & 89 & 1,53 & 2,45 & 37 & 8 \\
\hline BA $44-72$ & 570 & 90 & 340 & Franco-argilo-arenoso & 0 & 100 & 1,44 & 2,49 & 42 & 6 \\
\hline Bw1 $72-119$ & 560 & 80 & 360 & Argilo-arenoso & 0 & 100 & 1,30 & 2,55 & 49 & 3 \\
\hline Bw2 $119-150+$ & 540 & 90 & 370 & Argilo-arenoso & 0 & 100 & 1,40 & 2,57 & 45 & 3 \\
\hline \multicolumn{11}{|c|}{ PERFIL 5B - sucessão pasto/soja/cana não fertirrigada } \\
\hline A $0-29$ & 570 & 90 & 340 & Franco-argilo-arenoso & 10 & 76 & 1,83 & 2,55 & 28 & 14 \\
\hline AB $29-56$ & 570 & 90 & 340 & Franco-argilo-arenoso & 12 & 67 & 1,42 & 2,39 & 40 & 9 \\
\hline BA $56-73$ & 550 & 100 & 350 & Franco-argilo-arenoso & 2 & 95 & 1,46 & 2,50 & 41 & 6 \\
\hline Bw1 $73-97$ & 540 & 90 & 370 & Argilo-arenoso & 0 & 100 & 1,28 & 2,53 & 49 & 3 \\
\hline Bw2 $97-150+$ & 540 & 90 & 370 & Argilo-arenoso & 0 & 100 & 1,37 & 2,55 & 46 & 2 \\
\hline \multicolumn{11}{|c|}{ PERFIL 8C - vegetação natural } \\
\hline A $0-13$ & 570 & 90 & 340 & Franco-argilo-arenoso & 10 & 72 & 1,63 & 2,31 & 30 & 18 \\
\hline AB $13-41$ & 570 & 80 & 350 & Franco-argilo-arenoso & 10 & 76 & 1,49 & 2,35 & 37 & 10 \\
\hline BA $41-60$ & 550 & 100 & 350 & Franco-argilo-arenoso & 0 & 100 & 1,47 & 2,42 & 39 & 6 \\
\hline Bw1 $60-88$ & 540 & 90 & 370 & Argilo-arenoso & 2 & 95 & 1,53 & 2,48 & 38 & 3 \\
\hline Bw2 $88-150+$ & 540 & 90 & 370 & Argilo-arenoso & 4 & 91 & 1,53 & 2,55 & 40 & 3 \\
\hline
\end{tabular}


Em relação à porosidade total (PT), ocorre aumento em profundidade em todos os perfis, como esperado, sendo que no perfil 8C nota-se maior homogeneidade entre os valores. Nos horizontes superficiais A, dos perfis 3B, 4B e 5B, observam-se valores próximos a 30\% de PT. Não foi observada uma redução acentuada na PT dos perfis cultivados em relação ao perfil referência, o que faz supor que a relação entre macro e microporos pode estar sendo alterada, como é típico de horizontes compactados (Dias Júnior, 2000).

Os valores de $\mathrm{MO}$ mais elevados encontram-se nos horizontes A e $\mathrm{AB}$, decrescendo em direção aos $\mathrm{Bw}$, característica comum desses solos, como constatado em todos os perfis. Porém, observa-se que, nos perfis cultivados com cana, os valores de MO são menores do que no perfil de referência, 8C. Os menores valores de $\mathrm{MO}$ são encontrados nos horizontes A e AB do perfil 4B (10 $\left.\mathrm{g} \mathrm{cm}^{-3}\right)$. Observa-se, portanto, redução nos valores de MO dos solos cultivados, ainda que estes ocorram em manejo sob cultivo em resíduos de palhada da cana-de-açúcar, o qual tende a preservar a $\mathrm{MO}$ nos horizontes superficiais, mesmo se tratando do primeiro ciclo e do $4^{\circ}$ corte da cana. Contudo, corrigindo esses valores para o C orgânico, pode-se concluir que os teores não são elevados. Vale ressaltar que esses perfis não foram fertirrigados com vinhaça, uma vez que esses solos encontram- -se fora do raio de fertirrigação das usinas.

Em síntese, os atributos físicos e de MO apresentam evidências de degradação dos solos, marcados pelos valores elevados de ADA em contraposição aos valores reduzidos de GF, nos horizontes superficiais A e AB. É sabido que a dispersão de argila pode acarretar a eluviação e migração (translocação) de material para os horizontes inferiores dos solos, levando ao encrostamento superficial por adensamento. $\mathrm{O}$ aumento da Ds nos horizontes superficiais A e AB dos perfis 4B e 5B sugere isso. Apenas para o horizonte A de todos os perfis foi observada baixa PT, indicativa de restrições à infiltração de água, penetração de raízes e de trocas de calor e de gases com a atmosfera.

Quanto aos valores de RP, os resultados indicam que os perfis 4B e $5 \mathrm{~B}$ apresentaram as maiores resistências, com valores próximos a $6 \mathrm{MPa}$, considerados limitantes à penetração de raízes, variando em profundidade entre 0 e $10 \mathrm{~cm}$ para o perfil $4 \mathrm{~B}$ e entre 10 e $15 \mathrm{~cm}$ para o 5B, conforme se observa na Figura 1 (Silva et al., 2011). 


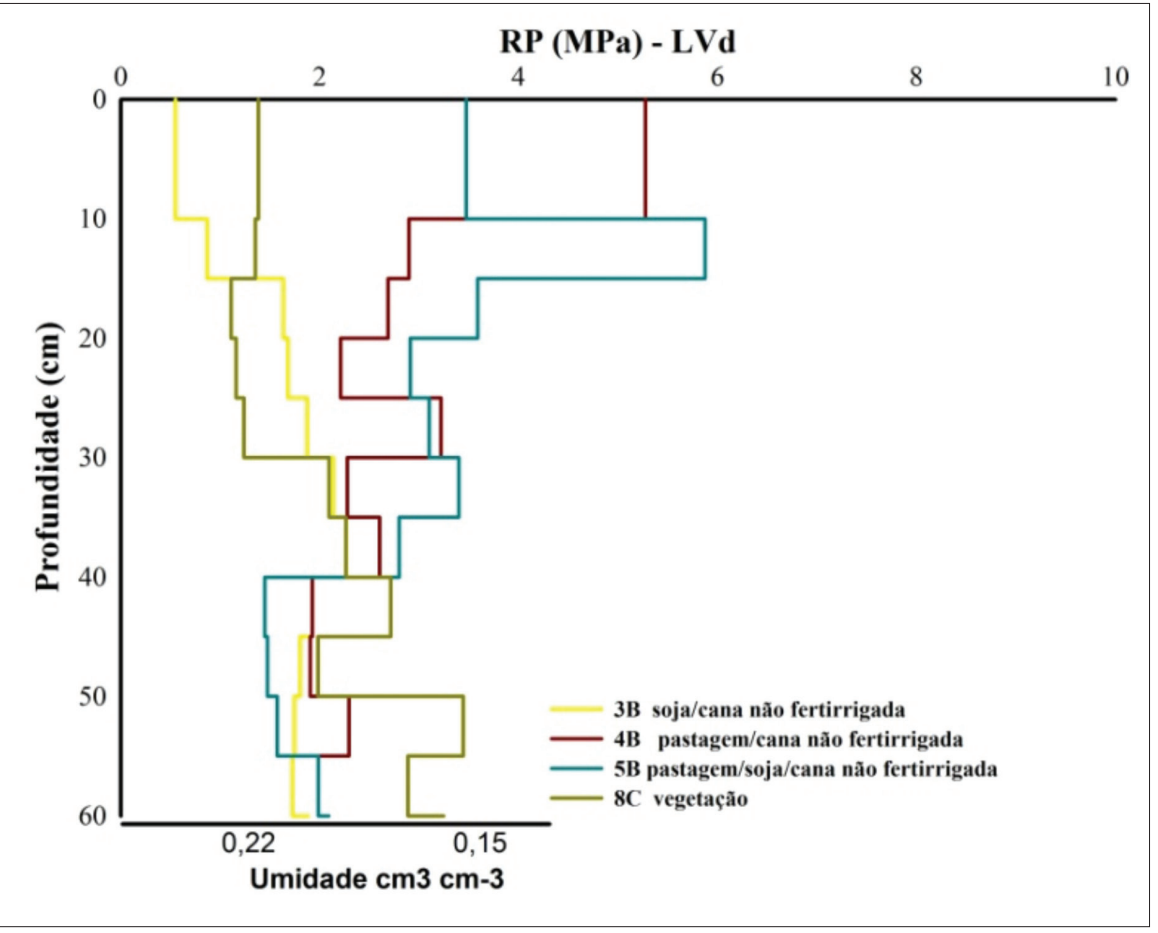

Figura 1 - Valores de RP em profundidade nos Latossolos Vermelhos distróficos.

Os valores de RP variam em relação à umidade, ao conteúdo de $\mathrm{MO}$ e de argila e à Ds; entretanto, valores maiores do que 2,5 a 3 MPa são, em geral, considerados indicadores de compactação. Assim, os perfis 3B e 8C não apresentaram valores de RP que evidenciem compactação, ao contrário dos demais, 4B e 5B, que apresentaram resistência até cerca de 30 cm, profundidade compatível com o efeito oriundo de máquinas pesadas utilizadas nos tratos agrícolas, sobretudo na colheita mecanizada, como já exposto.

Esse potencial de compactação ocorre mesmo se tratando de solos de textura média, indicando processos de degradação, uma vez que no geral esses solos apresentam maior capacidade de suporte de carga, em razão das frações grosseiras que podem aumentar as forças de atrito e assim dissipar a energia (Oliveira; Vaz; Reichardt, 1995; Vaz et al., 2002; Roque et al., 2010). 


\section{Indicadores micromorfológicos}

A Figura 2 apresenta o balanço dos constituintes da matriz do solo com base na observação microscópica de amostras em lâminas delgadas. A matriz dos LVd é composta, em geral, por um teor de material grosseiro maior do que de material fino, com comportamento das curvas relativamente paralelo. Em solos de textura média, a fração grosseira é constituída por grãos de quartzo de tamanho médio a pequeno e, raramente, por nódulos de ferro (EMBRAPA, 2006), como ora observado. O material fino apresenta menores teores no horizonte A dos perfis 3B e 8C, com cerca de 15 a $20 \%$, e aumenta nos horizontes $\mathrm{AB}$ chegando a $30 \%$, voltando a reduzir no BA e Bw1 para 20\%, aumentando ligeiramente no Bw2 (25\%). Os perfis $4 \mathrm{~B}$ e $5 \mathrm{~B}$ apresentam os valores mais elevados de material fino nos horizontes $\mathrm{A}$ e $\mathrm{AB}(\sim 35 \%)$, permanecendo constantes no perfil $5 \mathrm{~B}$ e reduzindo nos horizontes BA e Bw1 do perfil 4B (20\%), com aumento no Bw2 (30\%). Pode-se constatar um comportamento inverso das curvas de finos e poros, o que leva a supor possível perda de finos por lixiviação/ lessivagem superficial.

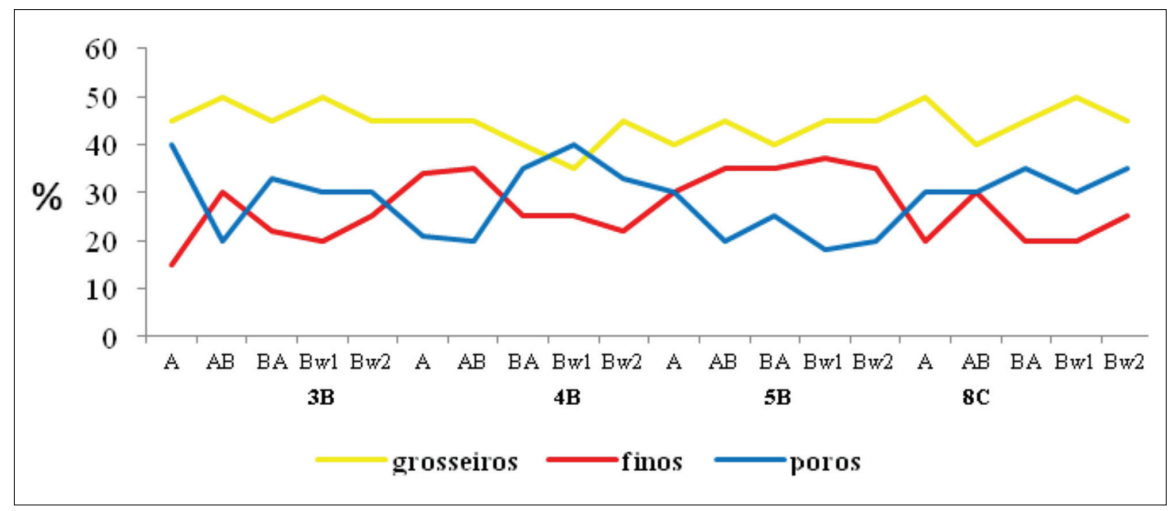

Figura 2 - Distribuição dos componentes grosseiros, finos e poros do fundo matricial das lâminas delgadas dos solos LVd.

De acordo com a Figura 2, os poros apresentam percentuais de concentração elevados no horizonte A, do perfil 3B ( $\sim 40 \%)$, que reduzem no AB (20\%) e voltam a aumentar nos demais horizontes. O perfil 4B também 
apresenta porosidade reduzida nos horizontes A e AB - cerca de $20 \%$-, aumenta nos demais horizontes, chegando a $45 \%$ de poros no horizonte Bw1. O perfil 5B apresenta cerca de 30\% de porosidade no horizonte A, é reduzida para $20 \%$ no $\mathrm{AB}, 25 \%$ no $\mathrm{BA}$ e $20 \%$ nos $\mathrm{Bw}$. O perfil $8 \mathrm{C}$ apresenta porosidade variando em torno de 30 a $35 \%$, sendo relativamente homogênea em todos os horizontes. As variações encontradas podem estar associadas ao manejo tecnificado da cana-de-açúcar. Porém, as diferenças entre finos e poros podem ser intrínsecas aos solos (EMBRAPA, 2006) ou mesmo herdadas de usos e manejos anteriores, posto que a cana é plantada muito recentemente ali (desde 2008).

A distribuição relativa entre as frações fina e grosseira dos solos LVd (Figura 3) apresenta estes três tipos distintos: enáulica (microagregada), gefúrica (pontes ligando grãos grosseiros) e porfírica (grosseiros cimentados por finos), além de rara distribuição quitônica (películas de finos envolvendo os grosseiros). Nos perfis 3B e 4B, predomina a distribuição dos tipos enáulica e gefúrica e, secundariamente, nas zonas adensadas, a distribuição porfírica, embora menos representativa no perfil 3B $(<20 \%)$ em comparação ao $4 \mathrm{~B}$, em que representa $50 \%$ no horizonte A e $30 \%$ no horizonte $\mathrm{AB}$. No perfil $5 \mathrm{~B}$, observa-se o predomínio da distribuição porfírica (60\%) nos horizontes A, AB, BA e até no Bw1.

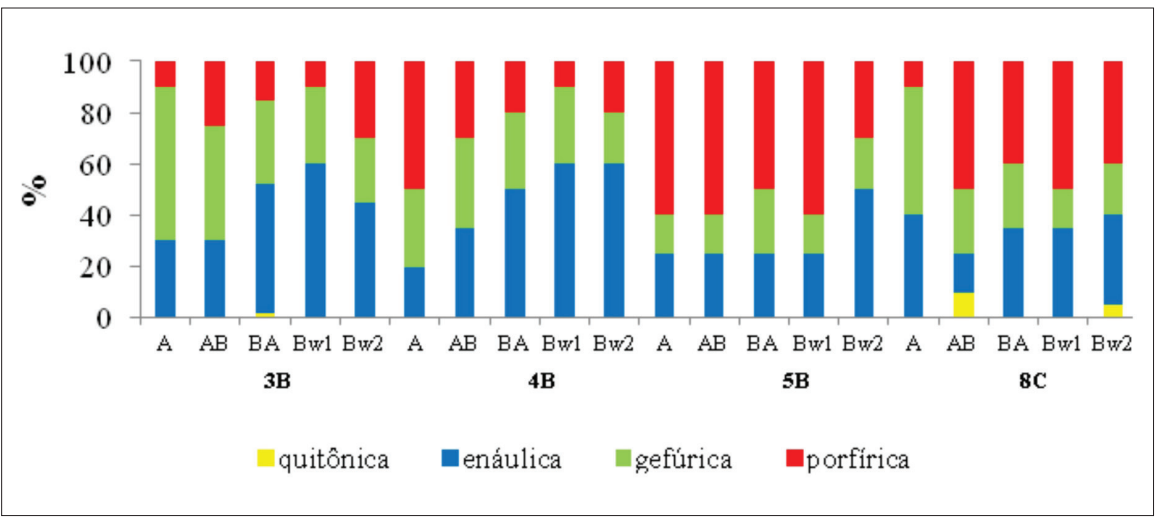

Figura 3 - Distribuição relativa dos horizontes.

A distribuição porfírica, sobretudo quando ocorre nos horizontes superficiais, costuma ser considerada indicadora de horizonte compactado, conforme observado por Silva et al. (1998), Soares et al. (2005), en- 
tre outros. O perfil 8C apresenta distribuição relativa gefúrica, enáulica e porfírica bem distribuídas entre si. Pontualmente, observou-se a distribuição relativa quitônica nos horizontes AB e Bw2 em cerca de 8\%.

Na Tabela 2, pode-se observar ainda que é possível distinguir três conjuntos de comportamento quanto à distribuição relativa nos perfis. $\mathrm{O}$ comportamento dos perfis $3 \mathrm{~B}$ e $4 \mathrm{~B}$ - que apresentam os horizontes A e $\mathrm{AB}$ com estrutura gefúrica associada à porfírica, evidentes no horizonte A do perfil 4B - está relacionado a uma fraca compactação. Já o perfil 5B apresenta predomínio de distribuição porfírica, nesse caso, relacionado aos processos mais intensos de compactação (Kertzman, 1996; Castro et al., 2003). O perfil 8C apresenta estrutura em blocos subarredondados e grumos pequenos, com pedalidade moderada, evidenciada por distribuição relativa enáulica-gefúrica, dominante em todos os horizontes; ainda que contenha pequenas zonas porfíricas adensadas, não foi considerado compactado. Esse perfil apresenta porosidade dominante intergrãos e interagregados médios a finos, representada por inúmeras cavidades milimétricas indicativas de boa atividade biológica em todo o perfil. Nos horizontes BA, dos perfis 3B e 4B, e nos Bw de todos os perfis com cana, predominam as distribuições enáulicas e gefúricas, relacionadas à microestrutura em blocos subarredondados pequenos de pedalidade forte a moderada. Na distribuição enáulica/gefúrica, o material fino encontra-se total ou parcialmente dissociado da fração grosseira, que é quartzosa fina. Mas, nas zonas adensadas porfíricas, as quais se encontram predominantemente nos horizontes $\mathrm{A}$ e $\mathrm{AB}$ do perfil $4 \mathrm{~B}$ e nos horizontes $\mathrm{A}, \mathrm{AB}, \mathrm{BA}$ e Bw1 do perfil 5B, a fração grosseira está cimentada pelos finos isotrópicos (Tabela 2).

O perfil 8C, de referência, ao contrário, apresenta uma distribuição constante ao longo de todos os horizontes, em que a atividade biológica evidenciada pela presença intensa de feições de preenchimento com diferentes graus indica que esses solos estão sendo biologicamente trabalhados, não se caracterizando como horizontes compactados. A distribuição enáulica é considerada padrão em Latossolos, sobretudo os argilosos e ricos em ferro e/ou alumínio, e indica boa evolução geoquímica e pedogenética do tipo ferralítica. As distribuições gefúrica e quitônica podem ser herdadas da rocha matriz ou resultar de processos de eluviação e perda de finos, especialmente em horizontes superficiais, como lembram Castro et al. (2003). Já a distribuição porfírica ocorre comumente em solos de 
textura média, com baixo teor de ferro, alumínio ou MO, e em horizontes saprolíticos, podendo ser herdada da rocha matriz; mas pode também ser provocada por compactação ou adensamento.

Tabela 2 - Caracterização micromorfológica do Latossolo Vermelho distrófico.

\begin{tabular}{|c|c|c|c|}
\hline $\begin{array}{l}\text { Horizonte } \\
\mathrm{Cm}\end{array}$ & $\begin{array}{l}\text { Microestrutura/ } \\
\text { pedalidade }\end{array}$ & $\begin{array}{l}\text { Porosidade (dominante): tipo } \\
\text { e dimensão }\end{array}$ & Feições: tipos (\%) \\
\hline \multicolumn{4}{|c|}{ PERFIL 3B- soja/cana } \\
\hline A $0-30$ & BSA mm, M a F & IG e IA, F; Cv e Ca mm & Nfe mm (1); Capmph (21) \\
\hline $\mathrm{AB} 30-70$ & BSA mm, M a F & IG e IA, F a MF; Cv e Fi & Capmph (20) \\
\hline BA $70-97$ & BSA mm, F a M & IG e IA, F a MF; Cv e Fi mm & Capmph (35) \\
\hline Bw1 $97-118$ & BSA mm, $\mathrm{F}$ a $\mathrm{M}$ & IG e IA, F a MF; Cv mm & Nca mm (1); Capmph (33) \\
\hline Bw2 $118-150+$ & BSA mm, F a M & IG e IA, F a MF; Cv e Ca mm & Capmph (30); Ex mm (1) \\
\hline \multicolumn{4}{|c|}{ PERFIL 4B - pasto/cana } \\
\hline A $0-20$ & BSA mm, M a F & IG e IA, F a MF; Ca e Fi mm & Capmph (20) \\
\hline AB $20-44$ & BSA mm, M a F & IG e IA, F a MF; Cv e Fi mm & Capmph (28) \\
\hline BA $44-72$ & BSA mm, F a M & IG e IA, F a MF; Cv e Fi mm & Capmph (35) \\
\hline Bw1 $72-119$ & BSA mm, F a M & IG e IA, F a MF; Cv e Cm mm & Capmph (36) \\
\hline Bw2 $119-150+$ & BSA mm, F a M & IG e IA, F a MF; Ca mm & Capmph (40) \\
\hline \multicolumn{4}{|c|}{ PERFIL 5B - sucessão pasto/soja/cana } \\
\hline A $0-29$ & BSA mm, M a F & IG e IA, F a MF; Cv e Fi mm & Nca mm (2); Capmph (20) \\
\hline $\mathrm{AB} 29-56$ & BSA mm, M a F & IG e IA, F a MF; Cv e Fi mm & Capmph (23) \\
\hline BA $56-73$ & BSA mm, M a F & IG e IA, F a MF; Cv e Ca mm & Capmph (23); Ex mm (1) \\
\hline Bw1 $73-97$ & BSA mm, M a F & IG e IA, F a MF; Cv e Fi mm & Capmph (26) \\
\hline Bw2 $97-150+$ & BSA mm, F a M & IG e IA, F a MF; Cv mm & Capmph (30) \\
\hline \multicolumn{4}{|c|}{ PERFIL 8C - vegetação natural } \\
\hline A $0-13$ & BSA e $\mathrm{G}$ mm, M & IG e IA, M a F; Cv e Ca mm & Capmph (17) \\
\hline AB $13-41$ & BSA e $\mathrm{G}$ mm, M a F & IG e IA, M a F; Cv, Ca e Fi mm & Capmph (20) \\
\hline BA $41-60$ & BSA e $\mathrm{G}$ mm, M a F & IG e IA, M a F; Cv e Ca mm & Capmph (23) \\
\hline Bw1 $60-88$ & BSA e $\mathrm{G}$ mm, M a F & IG e IA, M a F; Ca e Cm mm & Capmph (30) \\
\hline Bw2 $88-150+$ & BSA e $\mathrm{G}$ mm, M a F & IG e IA, M a F; Cv e Ca mm & Capmph (60) \\
\hline
\end{tabular}

Microestrutura/Pedalidade: BSA = blocos subarredondados, $\mathrm{G}=$ grumos, $\mathrm{F}=$ forte, $\mathrm{M}=$ moderada, $\mathrm{F}=$ fraca, $\mathrm{IA}=$ interagregados; Porosidade: $\mathrm{IG}=$ intergrãos, $\mathrm{IA}=$ interagregados, $\mathrm{Me}=$ média, $\mathrm{Fi}=$ fina, $\mathrm{MF}=$ muito fina, $\mathrm{Ca}=$ canais, $\mathrm{Cv}=$ cavidades, $\mathrm{Cm}=$ câmaras, $\mathrm{Fi}$ = fissuras; Feições: $\mathrm{NFe}=$ nódulos ferruginosos, $\mathrm{Nca}=$ nódulos de carvão, Capmph = canais preenchidos com material do próprio horizonte, Ex = excrementos.

A Figura 4 ilustra os resultados apresentados para os horizontes superficiais A e AB e subsuperficiais Bw1 e Bw2, em que os perfis 4B e 5B foram interpretados como de compactação moderada, pela redução da porosidade e formação de zonas adensadas de distribuição porfírica, e o perfil 3B interpretado como de compactação fraca, por apresentar tais características, porém em menor frequência e mais descontínuas. 

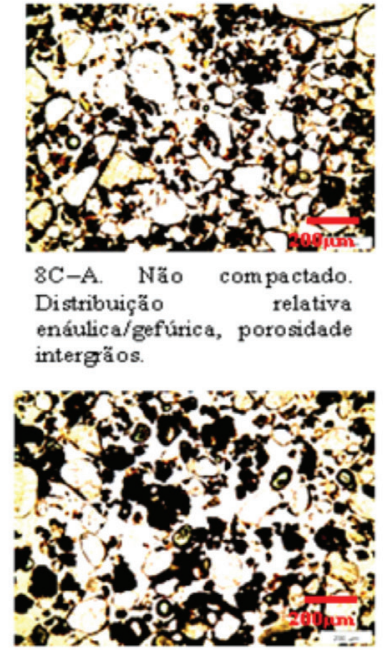

8C-Bw2. Não compactado. Distribuiçăo relativa enáulica/gefúrica, porosidade intergãos.

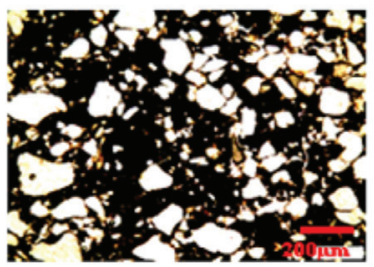

$$
\begin{aligned}
& 5 \mathrm{~B}-\mathrm{AB} . \quad \mathrm{Compactação} \\
& \text { modera da. Distribuiçăo } \\
& \text { relativa porfirica. }
\end{aligned}
$$

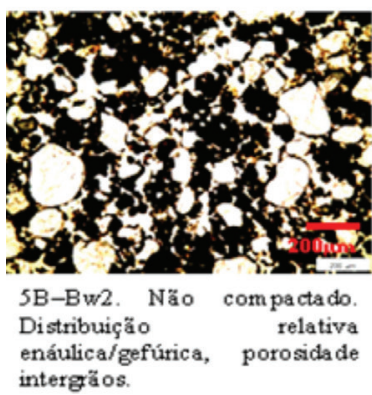

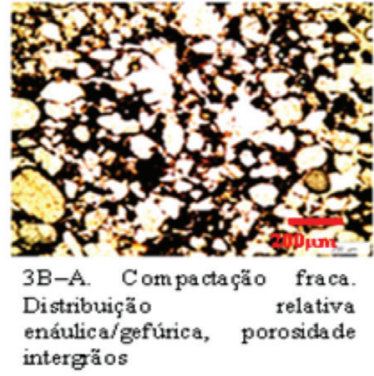

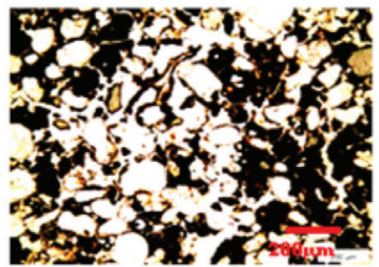

$3 \mathrm{~B}-\mathrm{Bw} 2$. Näo compactado.

Distribuiçäo relativa enáulica/gefúrica, porosidade intergãos.
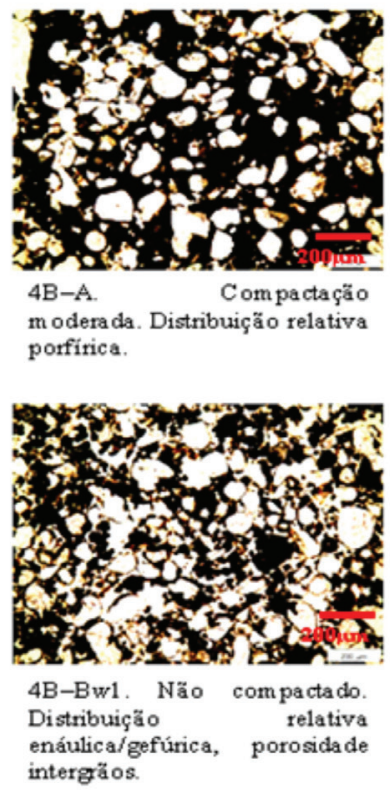

Figura 4 - Fotomicrografias das lâminas delgadas dos horizontes representativos dos perfis de LVd. Fração sólida corresponde à cor escura e os poros à clara (esbranquiçada). 
Em síntese, as modificações observadas referem-se à formação de zonas compactadas que afetaram principalmente os horizontes superiores dos solos (A e AB), sobretudo do perfil 5B, e foram associadas ao efeito do maquinário, que reduz a porosidade visível e instabiliza a estrutura dos solos, características corroboradas pelos demais dados relativos à morfologia dos solos em campo, aos valores de ADA, DS, consistência, sobretudo seca, e aos ensaios de RP. Os dados observados para os perfis cultivados com cana-de-açúcar, no entanto, quando comparados com os relativos ao perfil de controle (8C), não evidenciam grandes diferenças, o que remete ao fato de que a área de vegetação não estava plenamente preservada de interferências antrópicas.

\section{Considerações finais}

Os indicadores de qualidade física dos solos foram considerados satisfatórios por permitirem constatar sua instabilidade estrutural, relacionada ao processo de compactação, embora com diferentes graus para alguns horizontes do solo. Dentre os indicadores macromorfológicos, os que melhor ilustraram o processo foram: estrutura laminar, consistência seca, ADA, Ds e RP.

O indicador micromorfológico considerado mais relevante foi a distribuição relativa, marcada pelo predomínio de tramas porfíricas, que corroboram a interpretação de processo de compactação por cimentação do esqueleto pelo plasma. Indica, ainda, redução da porosidade, principalmente no perfil relativo à sucessão pasto/soja/cana.

Os processos de instabilidade estrutural por compactação, tal como observados, foram atribuídos ao manejo fortemente tecnificado da cana-de-açúcar, em razão do uso de maquinário pesado, visto que a compactação afeta os primeiros $15 \mathrm{~cm}$. Porém, de um modo geral, os indicadores obtidos permitem interpretar os processos de degradação estrutural dos solos como compatíveis com compactação moderada a fraca; até o momento, portanto, ainda seriam reversíveis com manejo adequado no preparo para o próximo ciclo.

\section{Agradecimentos}

Ao Conselho Nacional de Desenvolvimento Científico e Tecnológico (CNPq) e à Fundação de Amparo à Pesquisa do Estado de Goiás (FAPEG), 
pelo auxílio à pesquisa (Proc. $\mathrm{n}^{\circ}$ 479010/2009-9) e pela bolsa de formação (12/2009 a 08/2012), respectivamente.

\section{Referências}

BARBALHO, M. G. da S.; SILVA, A. A.; CASTRO, S. S. de. A expansão da área de cultivo da cana-de-açúcar na região sul do estado de Goiás de 2001 a 2011. Rev. Bras. de Ciências Ambientais, São Paulo, n. 29, p. 98-110, 2013.

CASAGRANDE, A. A. Compactação e manejo do solo na cultura da cana-deaçúcar. In: MORAES, M. H.; MULLER, M. M. L.; FOLONI, J. S. S. (Coords.). Qualidade física do solo: métodos de estudo - sistema de preparo e manejo do solo. Jaboticabal: Funep, 2002. p. 150-197.

CASTRO, S. S. Micromorfologia aplicada aos estudos de erosão. In: GUERRA, A. J. T.; BOTELHO, R. G. M.; SILVA, A. S. da (Orgs.). Erosão e conservação dos solos: conceitos, temas e aplicações, cap. $4^{\circ}$. Rio de Janeiro: Bertrand Brasil, 1999. p. 127-200.

CASTRO, S. S.; ABDALA, K; SILVA, A. A.; BORGES, V. M. S. A expansão da canade-açúcar no Cerrado e no Estado de Goiás: elementos para uma análise espacial do processo. Boletim Goiano de Geografia, Goiânia, v. 30, n. 1, p. 171-191, 2010.

CASTRO, S. S.; COOPER, M.; SANTOS, M. C.; VIDAL-TORRADO, P. Micromorfologia do solo: bases e aplicações. Tópicos em Ciência do Solo, v. 3, p. 107-164, 2003.

DIAS JÚNIOR, M. de S. Compactação do solo. Tópicos em Ciência do Solo, Viçosa, v. 1, p. 55-94, 2000.

EMBRAPA. Manual de métodos de análise de solos. 2. ed. Rio de Janeiro: EMBRAPA, 1997.

. Centro Nacional de Pesquisa de Solos. Sistema Brasileiro de Classificação de Solos. 2. ed. Rio de Janeiro: EMBRAPA, 2006.

KERTZMAN, F. F. Modificações na estrutura e no comportamento de um latossolo roxo provocadas pela compactação. 152 f. Tese (Doutorado) - Universidade de São Paulo, São Paulo, 1996.

LATRUBESSE, E. M.; CARVAlHO, T. M. de. Geomorfologia do Estado de Goiás e Distrito Federal. Secretaria de Indústria e Comércio, Superintendência de Geologia e Mineração. Goiânia: SIEG, 2006.

OLIVEIRA, G. C.; DIAS JÚNIOR, M. S.; RESCK, D. V. S.; CURI, N. Alterações estruturais e comportamento compressivo de um Latossolo Vermelho distrófico argiloso sob diferentes sistemas de uso e manejo. Pesquisa Agropecuária Brasileira, Brasília, v. 38, n. 2, p. 291-299, 2003.

OLIVEIRA, J. C. M.; VAZ, C. M. P.; REICHARDT, K. Efeito do cultivo contínuo da cana-de-açúcar em propriedades físicas de um Latossolo Vermelho escuro. Sc. Agrícola, Piracicaba, 52, n. 1, p. 50-55, jan./abr. 1995. 
PACHECO, E. P.; CANTALICE, J. R. B. Compressibilidade, resistência à penetração e intervalo hídrico ótimo de um Argissolo Amarelo cultivado com cana-de-açúcar nos tabuleiros costeiros de Alagoas. Revista Brasileira de Ciência do Solo, Viçosa, v. 35, p. 403-415, 2011.

PANKHURST, C. E.; MAGAREY, R. C.; STIRLING, G. R.; BLAIR, B. L.; BELL, M. J.; GARSIDE, A. L. Management practices to improve soil health and reduce the effects of detrimental soil biota associated with yield decline of sugarcane in Queensland, Australia. Soil \& Tillage Research, Amsterdam, v. 72, n. 2, p. 125137, 2003.

PEDROTTI, A.; MÉLLO JÚNIOR, A. V. Avanços em ciência do solo: a física do solo na produção agrícola e qualidade ambiental. São Cristovão: EdUFS, 2009.

REICHERT, J. M.; REINERT, D. J.; BRAIDA, J. A. Qualidade dos solos e sustentabilidade de sistemas agrícolas. Revista Ciência \& Ambiente, Santa Maria, v. 27, p. 29-48, 2003.

ROQUE, A. A. de; SOUZA, Z. M. de; BARBOSA, R. S.; SOUZA, G. de. Controle de tráfego agrícola e atributos físicos do solo em área cultivada com cana-de-açúcar. Pesquisa Agropecuária Brasileira, Brasília, v. 45, n. 7, p. 744-750, jul. 2010.

SANTOS, R. D. dos; LEMOS, R. C. de; SANTOS, H. G. dos; KER, J. C.; ANJOS, L. H. C. dos. Manual de descrição e coleta de solo no campo. Viçosa: EMBRAPA, 2005.

SCHNEIDER, P.; KLAMT, E.; GIASSON, E. Morfologia do solo: subsídio para caracterização e interpretação de solos a campo. Guaíba: Agrolivros, 2007.

SEVERIANO, E. da C.; OLIVEIRA, G. C. de; CURI, N.; DIAS JÚNIOR, M. de S. Solos cultivados com a cana-de-açúcar em Goianésia (GO). Revista Brasileira de Ciência do Solo, Viçosa, v. 33, p. 159-168, 2009.

SILVA, A. A.; CASTRO, S. S. Dinâmica de uso da terra e expansão da cana-deaçúcar entre os anos de 2004 e 2010, na microrregião de Quirinópolis, Goiás. In: PIETRAFESA, J. P.; SILVA, S. D. de (Orgs.). Transformações no Cerrado: progresso, consumo e natureza. Goiânia: Ed. PUC Goiás, 2011. p. 155-170.

SILVA, A. A.; SANT'ANA, G. R. S. de; BORGES, V. M. dos S.; CASTRO, S. S. de. Alterações físicas, químicas e morfológicas em um Latossolo Vermelho sob cultivo de cana-de-açúcar na microrregião de Quirinópolis, Goiás. In: CONGRESSO BRASILEIRO DE CIÊNCIA DO SOLO, 33. Anais... Uberlândia: SBCS, 2011. p. 1587-1592.

SILVA, A. J. N.; RIBEIRO, M. R.; MERMUT, A. R.; BENKE, M. B. Influência do cultivo contínuo da cana-de-açúcar em Latossolos Amarelos coesos do Estado de Alagoas: propriedades micromorfológicas. Revista Brasileira de Ciência do Solo, v. 22, p. 515-525, 1998.

SOARES, J. L. N.; ESPINDOLA, C. R.; FOLONI, L. L. Alteração física e morfológica em solos cultivados com citros e cana-de-açúcar, sob sistema tradicional de manejo. Ciência Rural, Santa Maria (RS), v. 35, n. 2, p. 353-359, 2005. 
STOLF, R.; FERNANDES, J.; FURLANI NETO, V. Recomendação para uso do penetrômetro de impacto modelo IAA/Planalsucar. Revista STAB - açúcar, álcool e subprodutos, v. 1, p. 18-23, 1983.

STOOPS, G. Guidelines for analysis and description of soil and regolith thin sections. Soil Science Society of America, Inc. Madison, Wisconsin, USA, 2003.

VAZ, C. M. P.; PRIMAVESI, O.; PATRIZZI, V. C.; IOSSI, M. de F. Influência da umidade na resistência do solo medida com penetrômetro de impacto. Comunicado Técnico, v. 51, São Carlos (SP): EMBRAPA Instrumentação Agropecuária, 2002. 5p.

Selma Simões de Castro - Licenciada em Geografia pela Universidade de São Paulo, Mestre em Geografia Física e Doutora em Ciências/Geografia Física pela mesma Instituição, Pós-Doutora em Ciência do Solo pelo INRA, Rennes (França), Professora titular de Geografia Física e Solos na Universidade Federal de Goiás.

Adriana Aparecida Silva - Licenciada e Bacharel em Geografia pela Universidade Federal de Goiás, Mestre e Doutora em Geografia pela mesma instituição, Professora na Universidade Estadual de Goiás, Unidade da Cidade de Goiás, Bolsista do Programa de Bolsa de Incentivo à Pesquisa e Produção Científica (PROBIP).

Recebido para publicação em 10 de maio de 2014

Aceito para publicação em 13 de junho de 2014 